\title{
el laborałorio en la investigación cerámica
}

\author{
F. ARREDONDO \\ ingeniero de caminos \\ andisis dilatométrico
}

\begin{abstract}
SINOPSIS
Se estudian las variaciones dimensionales que experimenta una arcilla durante su cocción, así como los métodos actuales para determinarlas. Se describe el dilatómetro, usado en el laboratorio de cerámica del I. T. C. C. para realizar el análisis dilatométrico.
\end{abstract}

Como hemos visto en nuestro artículo anterior (I), las arcillas sometidas a la acción del calor sufren una pérdida de peso, debido, principalmente, a la eliminación del agua -en sus diversas formas- y de clertas impurezas. asl como a ciertas reacciones que tienen lugar a determinadas temperaturas.

Simultănẹamente con esta pérdida de peso se observa una variación de dimensiones, que tiene gran importancia para identificar la arcilla y fijar la marcha de la cocción.

El procedimiento más elemental de determinar las variaciones dimensionales de una pieza de arcilla durante su cocción, es medir la pieza antes y después de someterla a la acción del calor, con objeto de hallar la diferencia.

Las variaciones dimenslonales así obtenidas, que se pueden Ilamar remanentes o definitivas, sólo pueden ser útiles para determinar las dimensiones que hay que dar a la boquilla de la galletera.

Esta diferencia de dimensiones no nos per-

(1) alnformes de fa Construtcián», núm. 85, noviembre de 1955. mite conocer la variación dimensional a lo largo de la cocción; para ello, es preciso conocer la historia de las dimensiones, en función de la temperatura, a fin de conocer los puntos o zonas criticas que son peligrosas desde ef punto de vista de la integridad del producto en curso de cocción.

La relación entre las variaciones dimensionales y la temperatúra puede representarse gráficamente, traducténdola en curvas características de cada tipo de arcilla. Las figuras 1,2 y 3 representan las variaciones dimensionales, según Munier, de la caolinlta, de la halósita y de la montmorillonita, respectivamente.

La marcha del horno se puede fijar sin previa determinación de la curva dilatométrica de una pasta cerámica; pero es expuesto, porque si se sigue una marcha lenta, buena por ser prudente, resulta antieconómica, y si se sigue una marcha rápida, económicamente más ventajosa, se incurre en el riesgo de no seguir el ritmo que exigen las complejas transformaciones de la arcilla y producir la rotura de la pieza.

Las causas principales de los cambios dimensionales son: la liberación brusca de masas gaseosas en el interior del material, las modificaclones alotrópicas y las diferencias de dilatación o retracción entre dos puntos de la pieza.

Como no conocemos el módulo de elasticidad del material durante la cocción, no se pueden calcular las tensiones ocasionadas por un cambio dimensional; por tanto, no podemos regular el valor de dichas tensiones para evitar la rotura, lo que exigiria conocer el valor de la tensión correspondiente a ésta. Como tampoco 
conocemos la velocidad desarrollada para alcanzar el equilibrio de temperaturas entre las partes más y menos calientes de la pieza, nos hallamos ante la obligación de llegar a una solución intermedia, en la que la cocción sea buena, nales de una arcilla, se ha expuesto ya en otro lugar (2).

Las curvas de cambios dimensionales se han determinado empleando unos aparatos llamados dilatómetros.

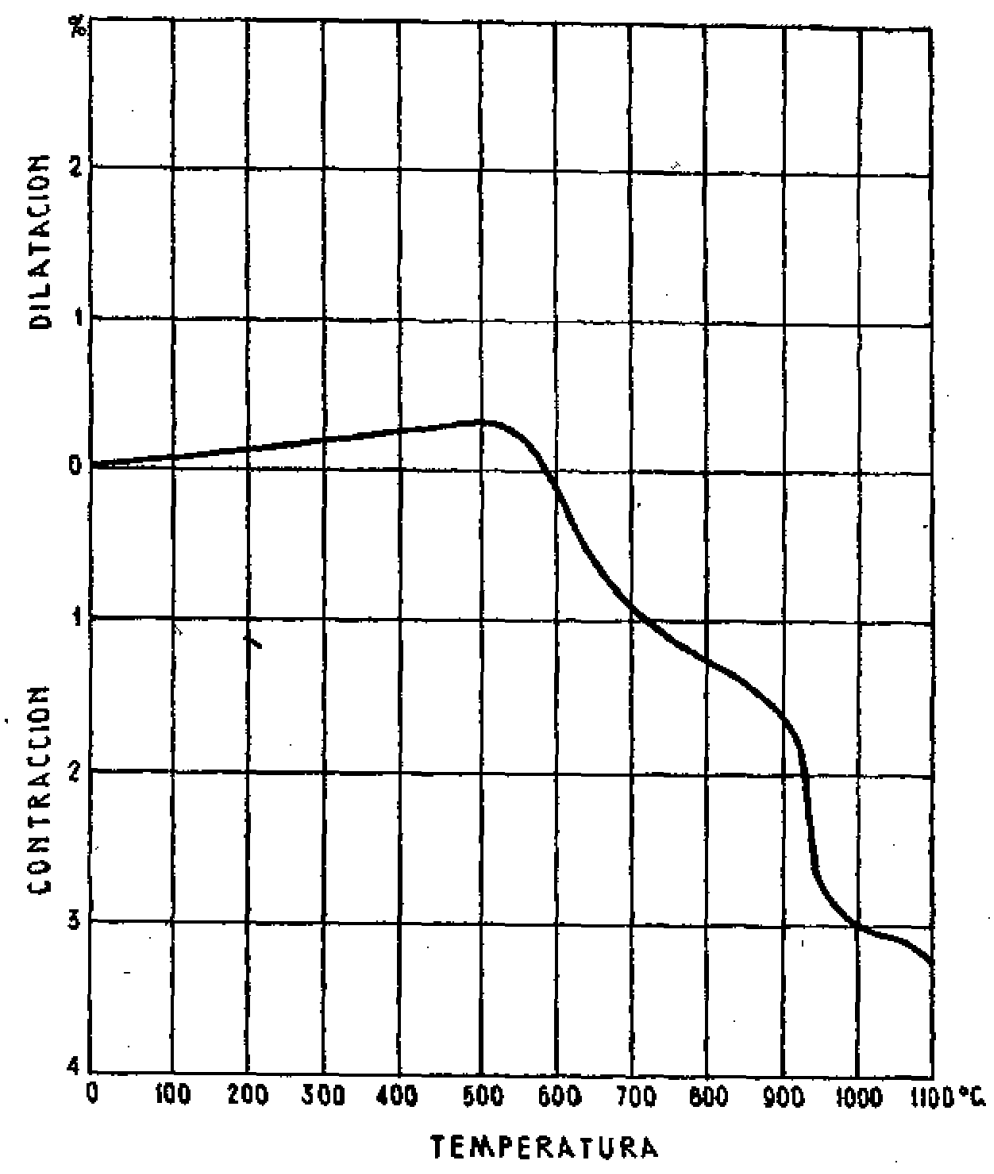

Fig. 1

tanto desde el punto de vista técnico como económico.

La solución sencilla que suele adoptarse, dentro de límites de errores aceptables, consiste en dar a la cocción una marcha tal, que las variaciones dimensionales sean proporcionales al tiempo.

La determinación de una curva de cocción, partiendo de la curva de variaciones dimensio-
Uno de los tipos más sencillos de estos aparatos, concebido por Miehr al principio de la utilización de esta técnica, permite transmitir la dilatación de la probeta, mediante una varilla, a un amplificador de palanca.

Al someter este dispositivo a la acción del calor, la probeta y la varilla se dilatan simultá.

(2) aArcillas y ladrillosw, Il parte. Instituto Tócnico de la Construcción y del Cemento, octubre do 1951 (del mismo autor). 
neamente. Este procedimiento es poco preciso, porque a la dilatación de la varilla se suma la de la probeta.

Este error desaparace en los dilatómetros diferenciales, de los que poseemos en nuestro la boratorio de cerámica uno de los modelos más a una placa fotográfica, en la que queda impresa una curva del tipo de la representada en la figura 5.

Por construcción, el ángulo $X_{p}, Y$, del esquema de la figura 6 , es recto, $y$ el eje $p_{1} X$ es horizontal.

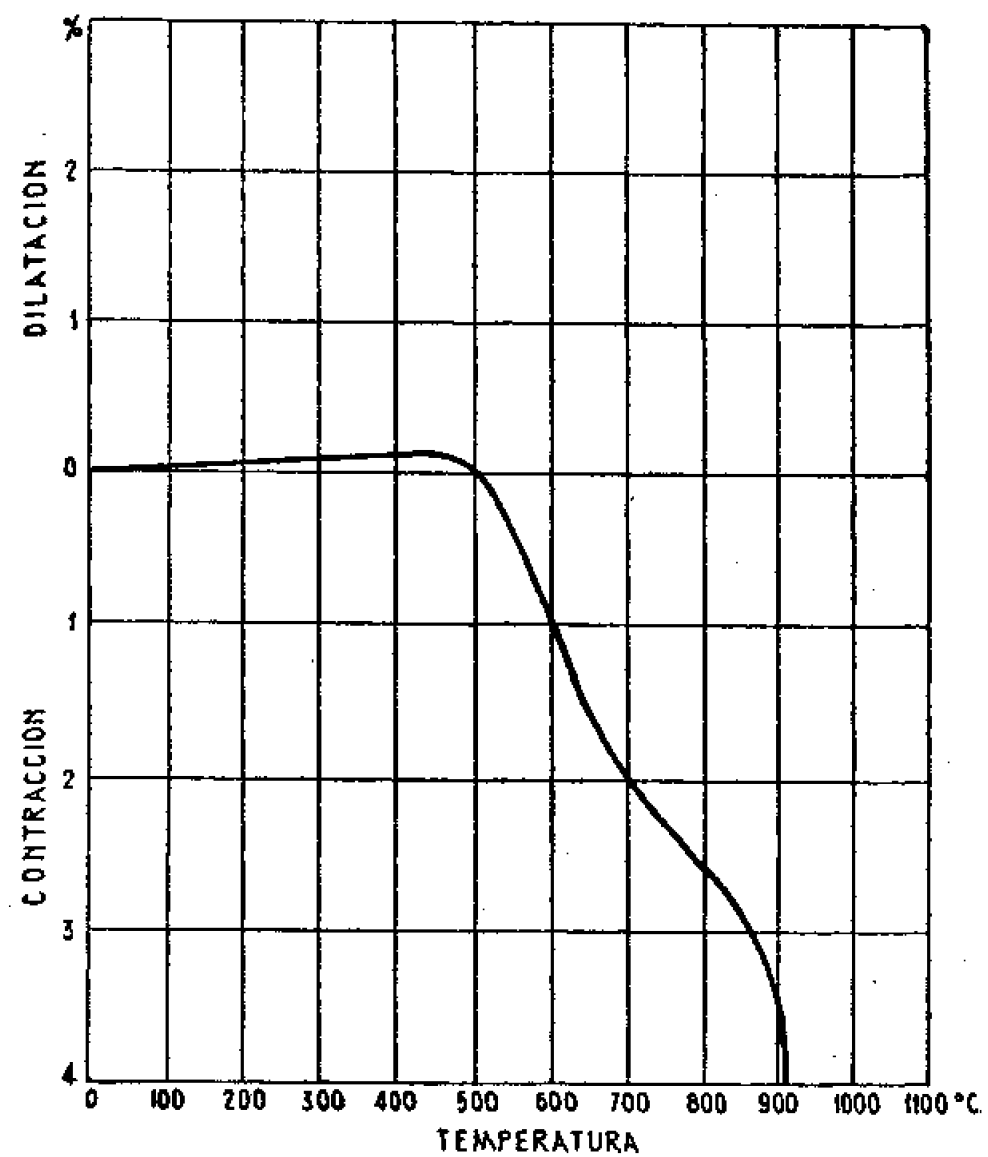

Fig. 2

perfeccionados. Este modelo es un dilatómetro Cheveniard, cuyo conjunto puede verse en la figura 4.

En el interior de un horno regulado automáticamente, y cuyo programa de calentamiento ha sido fijado, se coloca la muestra que se ensaya y otra patrón, metálica, cuya dilatación se conoce perfectamente. Las dilataciones de la muestra y del patrón se transmiten, mediante unas varillas, a una palanca óptica dotada de un espejo que, por reflexión, dirige el rayo de luz
Al elevarse la temperatura del horno, el espejo sufre una doble desviación alrededor de los ejes $p_{3} X$ y $p_{1} Y$. La abscisa $(x)$ de un punto de la curva descrita es proporcional a la dilatación del patrón, y la ordenada (y)́ lo es a la diferencla de las dilataciones entre la probeta de arcilla y la muestra patrón, puesto que las dilataciones en las dos varillas iguales son equivalentes, anulándose, por tanto, al efectuar la diferencla.

Como conocemos la dilatación de la muestra patrón, por un ensayo previo, podemos deter- 
minar fácilmente la dilatación absoluta de la arcilla en función de la temperatura.

Para evitar el trabajo de gabinete, posterior a la obtención de la curva diferencial, se han ideado otros dilatómetros, llamados absolutos, basados en métodos ópticos. Su fundamento con- a la probeta, y no a los órganos del aparato. También ha observado que todo dispositivo de medida, en el que los elementos de transmisión de la dilatación tengan su eje paralelo al eje longitudinal de la probeta, no puede dar medidas absolutas. Por el contrario, si los ejes de

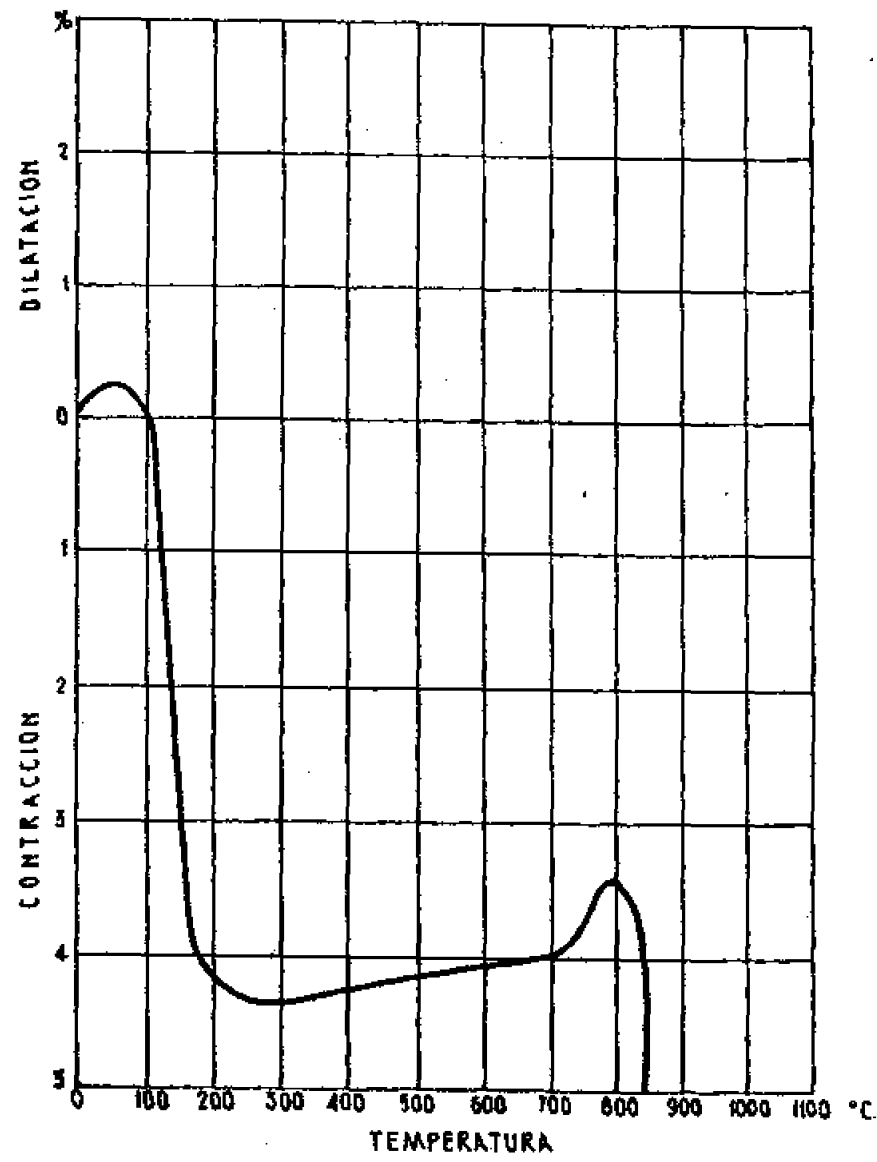

Fi. 3

siste en observar, durante el calentamiento, una probeta provista de dos referencias. Las deformaciones así observadas son absolutas, ya que se miden directamente.

Desgraciadamente, hasta el momento presente, no se ha podido lograr que estos dilatómetros sean registradores, lo que complica el trabajo.

A. Baudran, de la Société Françaișe de Céramique, ha estudiado detenidamente este tema, buscando un esquema tal de dilatómetro, que la medida de la dilatación se refiera exclusivamente los elementos de transmisión son perpendiculares al eje longitudinal de la probeta, y si su separación es igual a la longitud de ésta, los desplazamientos de los ejes son únicamente función de los cambios dimensionales de la probeta.

En el Congrès Technique Céramique, celebrado en París, en junio de 1955, se ha presentado un nuevo tipo de dilatómetro absoluto registrador, del que se da un esquema en la figura 7 , pero que presenta bastante complicación para registrar las temperaturas y deformaciones. 


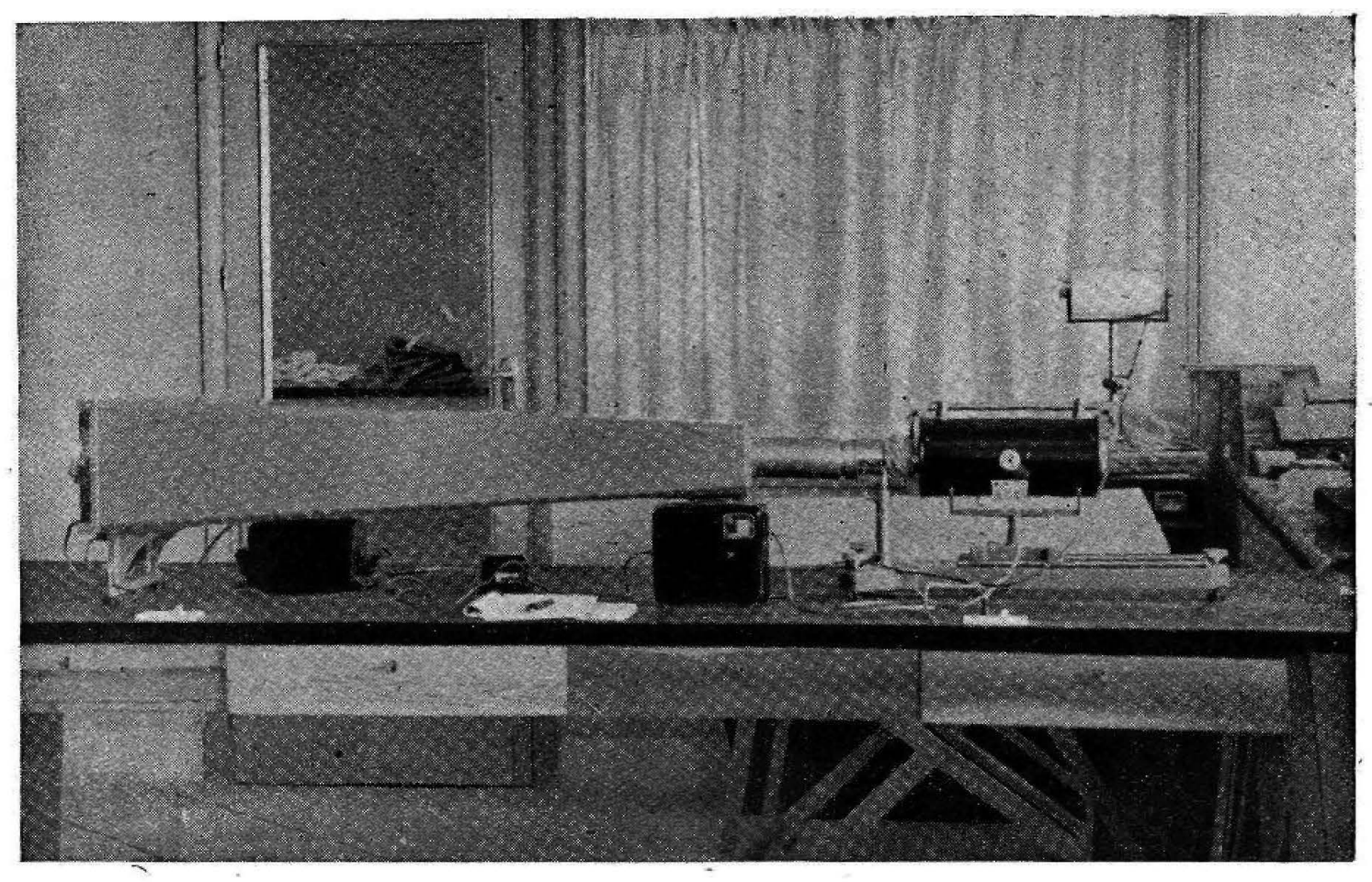

Fig. 4

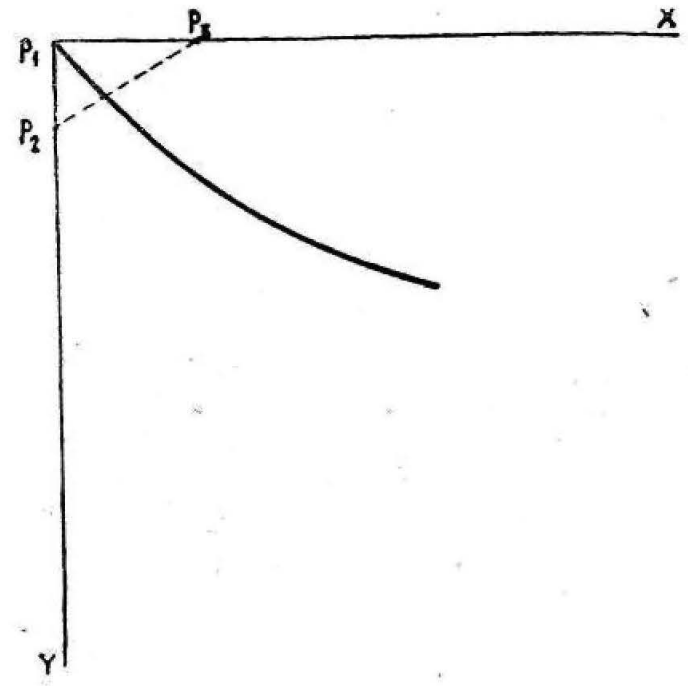

Fig. 5

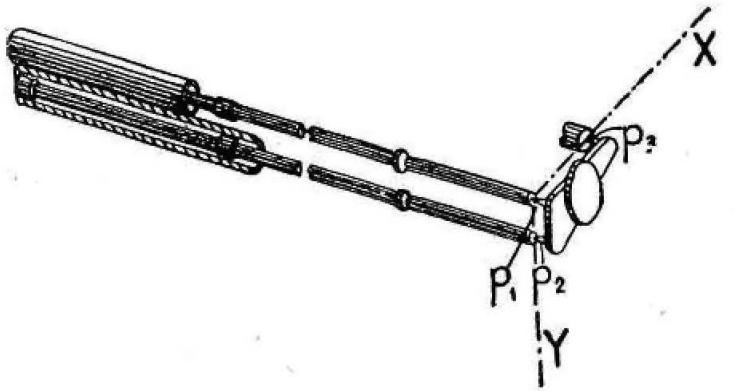

Fig. 6



Fig. 7 


\section{andisis tormogravimetrico}

\section{SINOPSIS}

Se estudian las variaciones de peso que experimenta una arcilla durante la cocción y los métodos que existen para determinarlas. Se describe la termobalanza, usada en el laboratorio de cerámica del Instituto Técnico de la Construcción y del Cemento para realizar el análissis térmico ponderal.

El Instituto Técnico de la Construcción y del Cemento ha ampliado sus instalaciones de la División de Materiales, al poner en funcionamiento un laboratorio de cerámica. Se Itena de este modo una necesidad sentida hace tiempo, ya que la cerámica de construcción, que tiene un uso amplísimo en nuestro país, está, hasta ahora, basada en un completo empirismo.

La mayor parte de las instalaciones están ya puestas a punto, y las restantes lo estarán en breve, incluso las que se refieren al control de secaderos y hornos industriales.

Este artículo es el primero de una serie, en los que se irán describiendo las posibilidades de dicho laboratorio.

La industria cerámica debe su existencia a las transformaciones que la arcilla experimenta al ser sometida a la acción del calor.

Si no existieran estas transformaciones, que hacen pasar la arcilla de una masa plástica y no resistente a un cuerpo endurecido y apto para soportar cargas, no existirla esta industria, de extensa tradición en nuestro país, ya que el barro sin cocer encuentra gran limitación en su uso y presenta propiedades poco aprovechables en la industria de la construcción.

Uno de los cambios primeramente observados al someter una arcilla a la acción del calor, es la variación de peso.
Esta variación de peso se debe, fundamentalmente, a la deshidratación de las arcillas, pero no en su totalidad, ya que, además, puede originarse por eliminación de ciertas impurezas, como es la materia orgánica, o por la descomposición de los carbonatos o por procesos de oxidación o reducción.

Todas las arcillas contlenen agua de constitución, agua de absorción y agua zeolítica. Todo el agua es eliminada mediante la acción del calor; pero el agua de cada uno de los tjpos citados se eltmina a una temperatura, o en una zona de temperaturas, determinada y característica para cada tipo de arcilla.

Las figuras 1,2 y 3 representan las curvas de deshidratación de la caolinita, montmorillonita e illita, respectivamente, según Longhnchambon.

Asi, pues, desde un punto de vista mlneralóglco, el conocimiento de la curva de deshidratacjón es de gran importancia para la identificación de la arcilla sometida a ensayo.

Pero, como hemos dicho anteriormente, las variaciones de peso no dependen exclusivamente del agua que pueda contener la arcilla en una u otra forma. La pasta cerámica puede contener otros elementos que experimenten variaciones de peso y, entonces, la curva variación de pesotemperatura será un dato interesante para fijar puntos o zonas críticas al establecer la curva de cocción de productos cerámicos en hornos industriales.

El fenómeno de la variación de peso en función de la temperatura puede representarse gráficamente, obteniéndose curvas que son características de cada arcilla, en cuanto a deshidratación se refiere.

Esta curva puede dibujarse por varios procedimientos: uno de ellos consiste en colocar en un horno tantos crisoles como valores de la temperatura vayamos a considerar. Cada crisol 


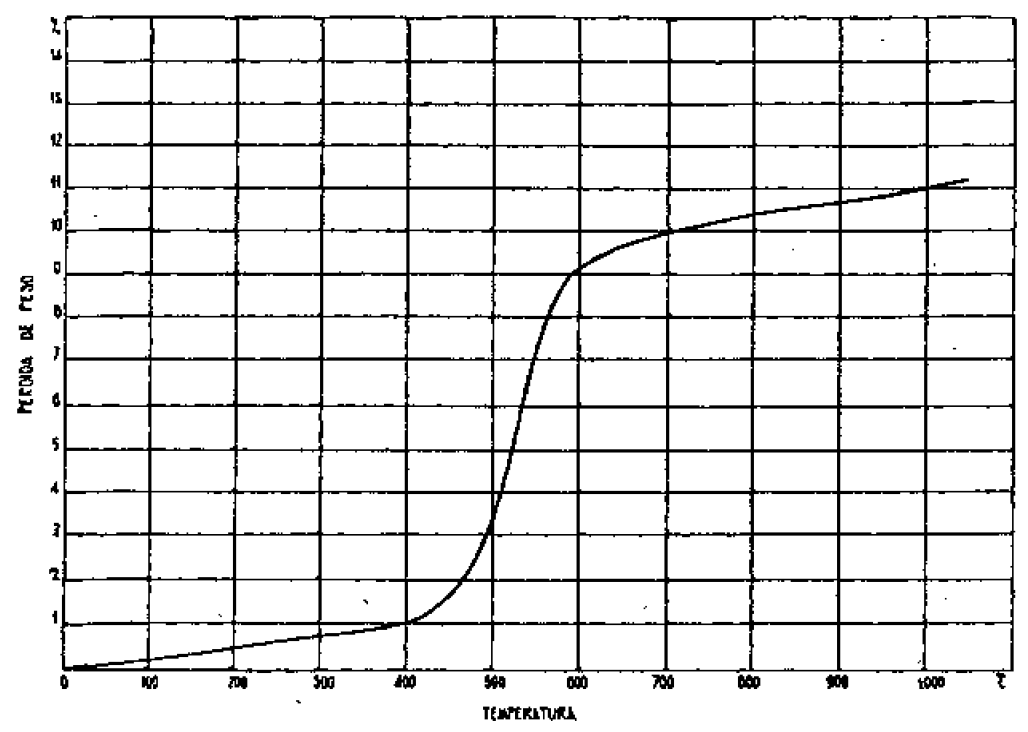

Fig. 1

contendrá un peso fijo de arcilla. Cuando el horno vaya alcanzando cada una de las temperaturas fijadas, se saca un crisol y se pesa. Cada pareja de números, temperatura, y pérdida de peso, será un punto de la curva en cuestión.
Este sistema no requiere ningún aparato especial $y$, por tanto, la curva es fácil de determinar. Pero tiene el inconveniente de que desconocemos lo que ha ocurrido en los intervalos entre cada dos temperaturas observadas, a menos que

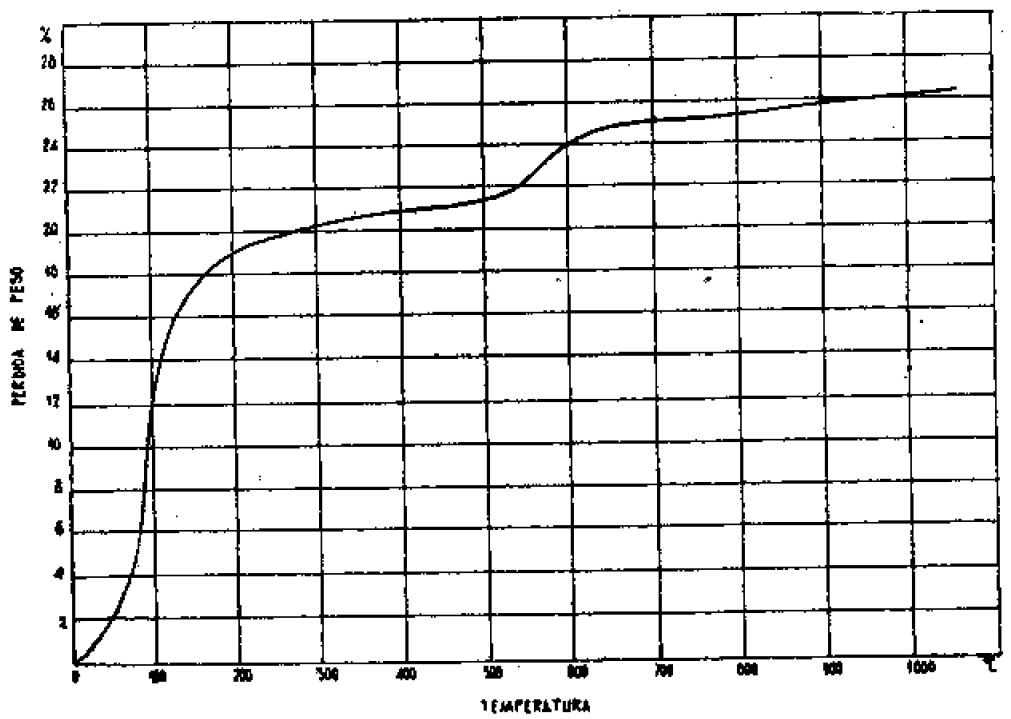

Fíg. 2 


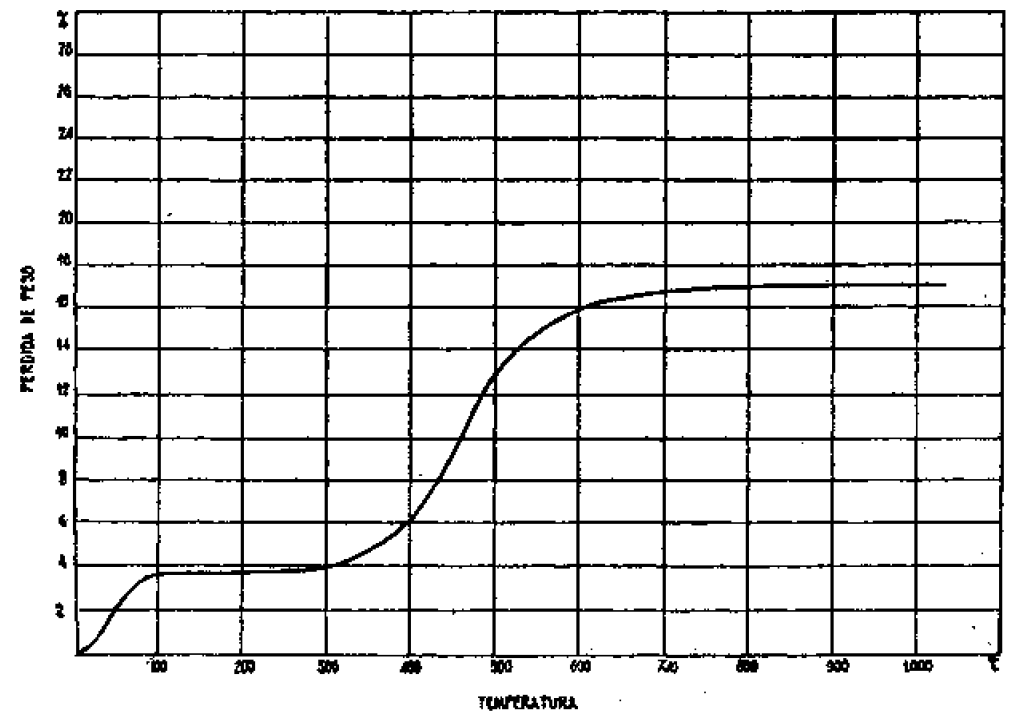

Fig. 3

estos intervalos sean muy pequeños, en cuyo caso la operación se complica requiriendo la atención casi constante de un operador mientras dura el ensayo.

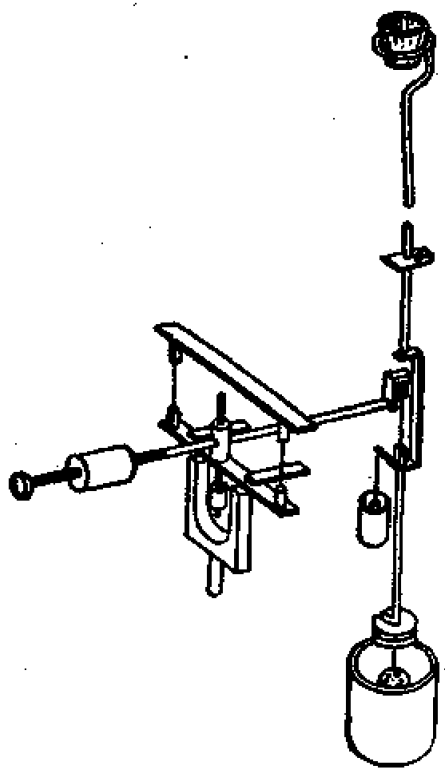

Fig. 4
. Es más preciso el método continuo, en el que se utiliza la termobalanza.

Existen diversos tipos de este aparato con características similares, pero adaptadas a las necesidades de cada caso particular.

En nuestro laboratorio utilizamos la termobalanza CHEVERNARD, cuyo esquema puede verse en la figura 4.

La arcilla sometida a estudio se coloca en el crisol situado en la extremidad superior de una varilla de sílice unida al brazo mayor de una balanza muy sensible. Dicha varilla penetra en un horno eléctrico de regulación automática, que sigue un programa de calefacción previsto y que alcanza $1.050^{\circ} \mathrm{C}$.

El brazo menor de la balanza lleva un espejo. que envia un rayo luminoso a un papel fotográfico enrollado en un tambor de movimiento constan. te. Cada variación de peso hace desviarse el espejo un ángulo proporcional a dicha variación; por ser este ángulo sumamente pequeño, la diferencia de ordenadas, correspondiente a una variación de peso dada, puede considerarse, con gran aproximación, proporcional a dicha variación de peso.

La figura 5 muestra el conjunto de la termobalanża con registro fotográfico.

También es posible adaptar a esta termobalanza un equipo de registro gráfico; en cuyo caso, 


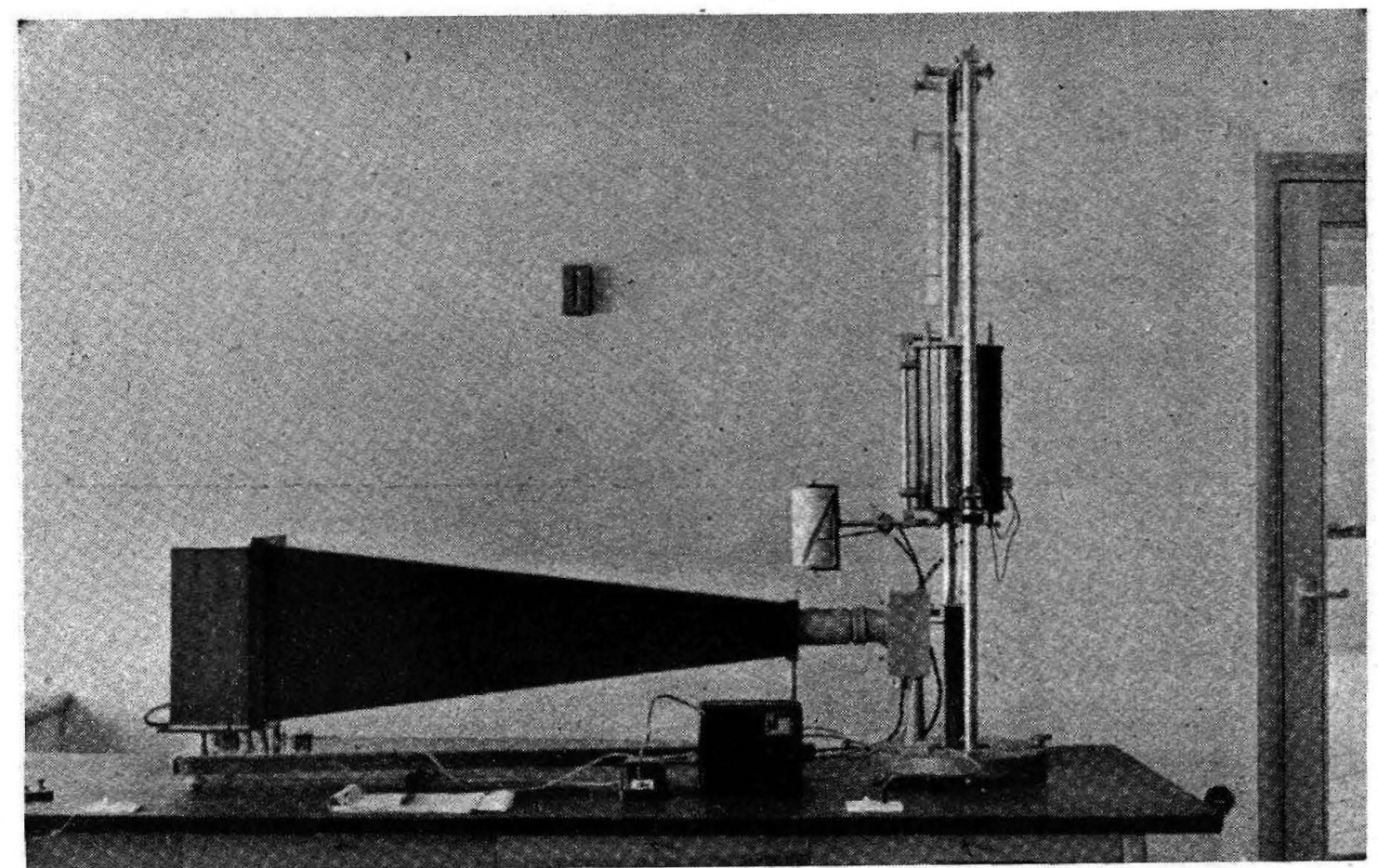

Fig. 5

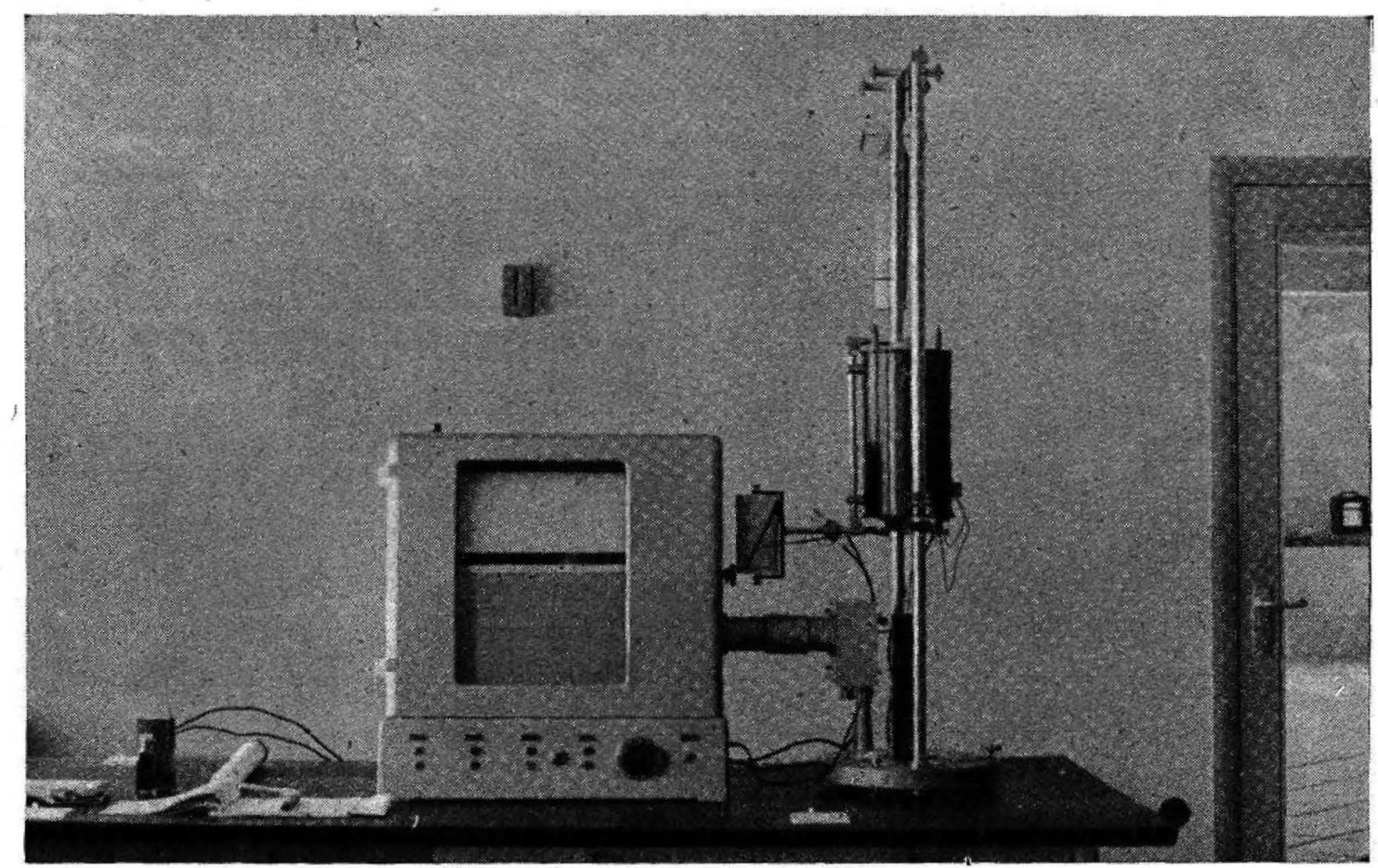

Fig. 6 
la variación de peso hace moverse a una aguja, la cual abre o cierra un circuito que actúa sobre el desplazamiento horizontal de una pluma que dibuja la curva correspondiente sobre un papel, en función del tiempo. Si la elevación de tem- cial, concebido por W. L. de Keyser. Con este aparato, en vez de inscribir la curva variación de peso-temperatura, $p=f(t)$, se registra la variación de la pendiente de dicha eurva, es decir, su derivada $\mathbf{d p} / \mathbf{d t}=\mathbf{f}^{\prime}(\mathbf{t})$, que, según $\mathrm{A}$. Lam-

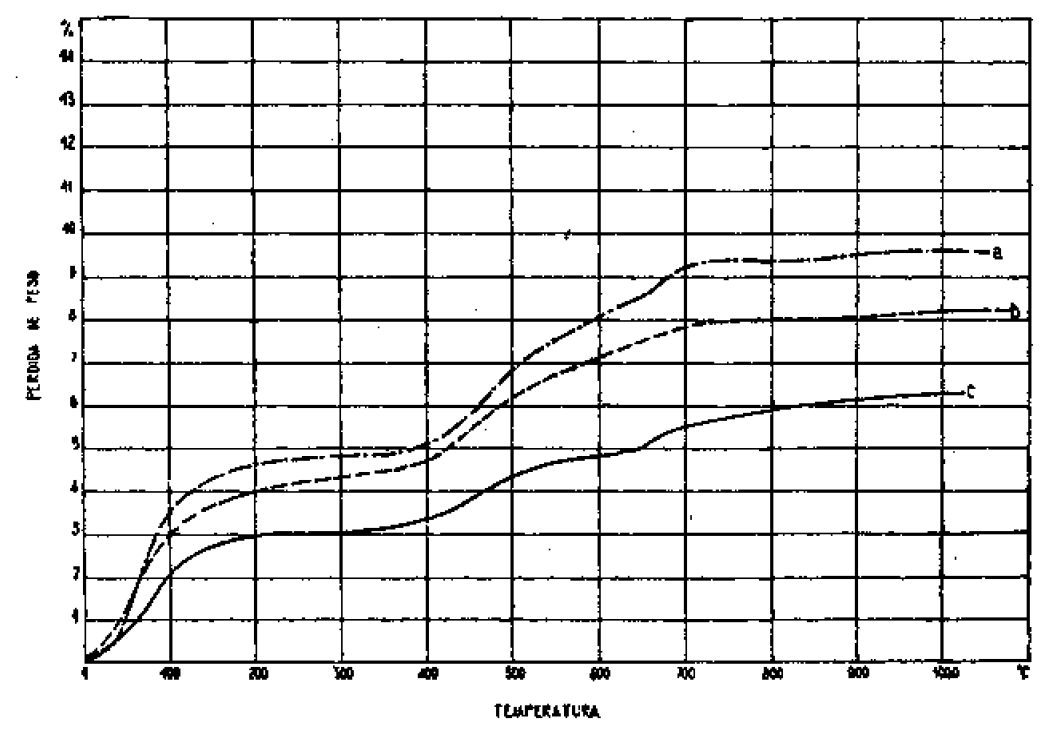

Fig. 7

peratura es función lineal del tiempo, puede sustituirse la escala de tiempos por la correspondiente de temperaturas, quedando las curvas en la forma usual.

La figura 6 nos muestra la termobalanza, con registro gráfico, en pleno funcionamiento.

Con el registro gráfico se tiene la ventaja de poder, en todo momento, seguir el curso del ensayo; no así el fotográfico, que nada permite saber del ensayo hasta terminado éste y revelado el papel.

La figura 7 representa una serie de curvas obtenidas en nuestro laboratorio. La curva a corresponde a una arcilla sola; la b, a la misma arcilla con un $20 \%$ de arena, y la c con un $40 \%$ de arena.

Existe otro tipo de termobalanza, la diferen- bert, es mucho más característica del producto analizado porque representa la velocidad de la variación del peso en función de la temperatura.

Keyser da como razón del uso de la termo. balanza diferencial la circunstancia de no haber podido obtener, con el registro fotográfico, diagramas de dimensiones grandes, hablendo tenido que recurrir a utilizar una balanza poco sensible o emplear cantidades de muestra muy reducidas, con que la precisión disminuye bastante.

Lambert ha puesto a punto una balanza de este tipo, que dibuja simultáneamente las dos curvas: la de variaciones de peso-temperatura y la de velocidad de la variación de peso-temperatura. 


\section{estudio del secado}

\section{SINOPSIS}

Se describe someramente el mecanismo del secado y su representación mediante las curvas de BIGOT, así como el aparato para su registro gráfico.

Una de las propiedades características de las arcillas, es la plasticidad que adquieren al ser amasadas con agua. Esta plasticidad permite darles cualquier forma en el periodo de moldeo.

Pero es de sobra sabido que antes de colocar estas piezas en el horno debe ser eliminada el agua. Esto da lugar al proceso de secado que, - sin duda, es el más delicado de toda la fabrlca. ción, $y$, por consiguiente, debe ser estudiado detenidamente.

Durante la eliminación del agua, según Bourry, se observan tres fenómenos sucesivos:

1.\%) La pasta disminuye de volumen proporcionalmente al agua eliminada.

2.0) Comienzan a formarse poros, pero la pasta sigue contrayéndose.

3.9) Al dejar de disminuir el volumen, los poros que se producen son proporcionales al agua eliminada.

Es decir, al aplicar calor a una arcilla recién moldeada, hay un doble efecto: pérdida de peso por una parte y contracción por otra.

El estudio de la relación de estas dos variables fué estudiado primeramente por Blgot, que la representó por una curva, llamada desde entonces curva de Bigot. Esta gráfica tiene el aire de la representada en la figura 1 . Como puede verse, esta curva confirma la hipótesis de Bourry.

Durante la primera parte del secado (eliminación del agua coloidal) existe siempre el peligro de fisuración $y$, por tanto, de rotura, debiéndose extremarse el cuidado al conducir el proceso.
Durante la segunda parte (eliminaclón def agua de interposición) la contracción ha cesado $y$, por consiguiente, el secado puede acelerarse sin miedo a roturas.

El estudio y determinación de la curva Bigot es, pues, de importancia industrial considerable, pues marca el momento que puede conduclrse el secado rápidamente y sin temor al fracaso.

La primera duda que se presenta para realizar este estudio, es la consistencia de la pastà.

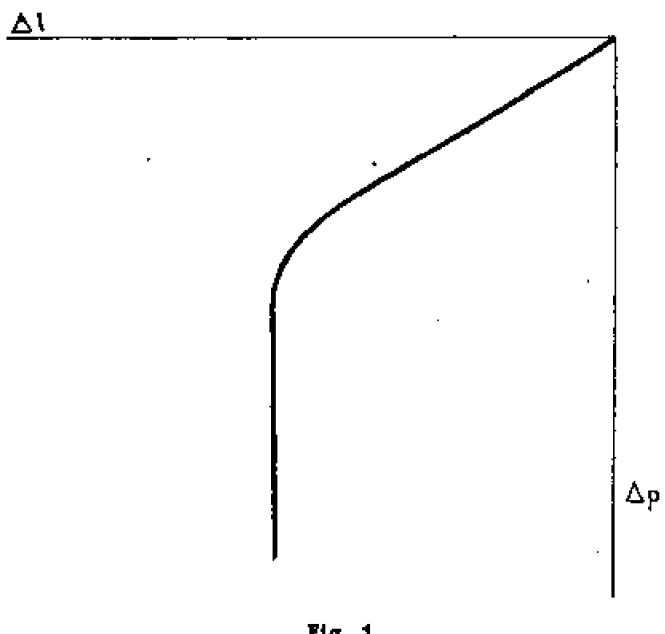

Fig. 1

En los laboratorios de la Société Française de Céramique se ha estudiado detenidamente este problema, haciéndose ensayos con pasta normal, es decir, amasada con el máximo de agua para que no se pegue a los dedos; con barbotina endurecida sobre molde de yeso; con arcilla fuertemente prensada, y con pasta obtenida en galleteras sin vacío y con él. 


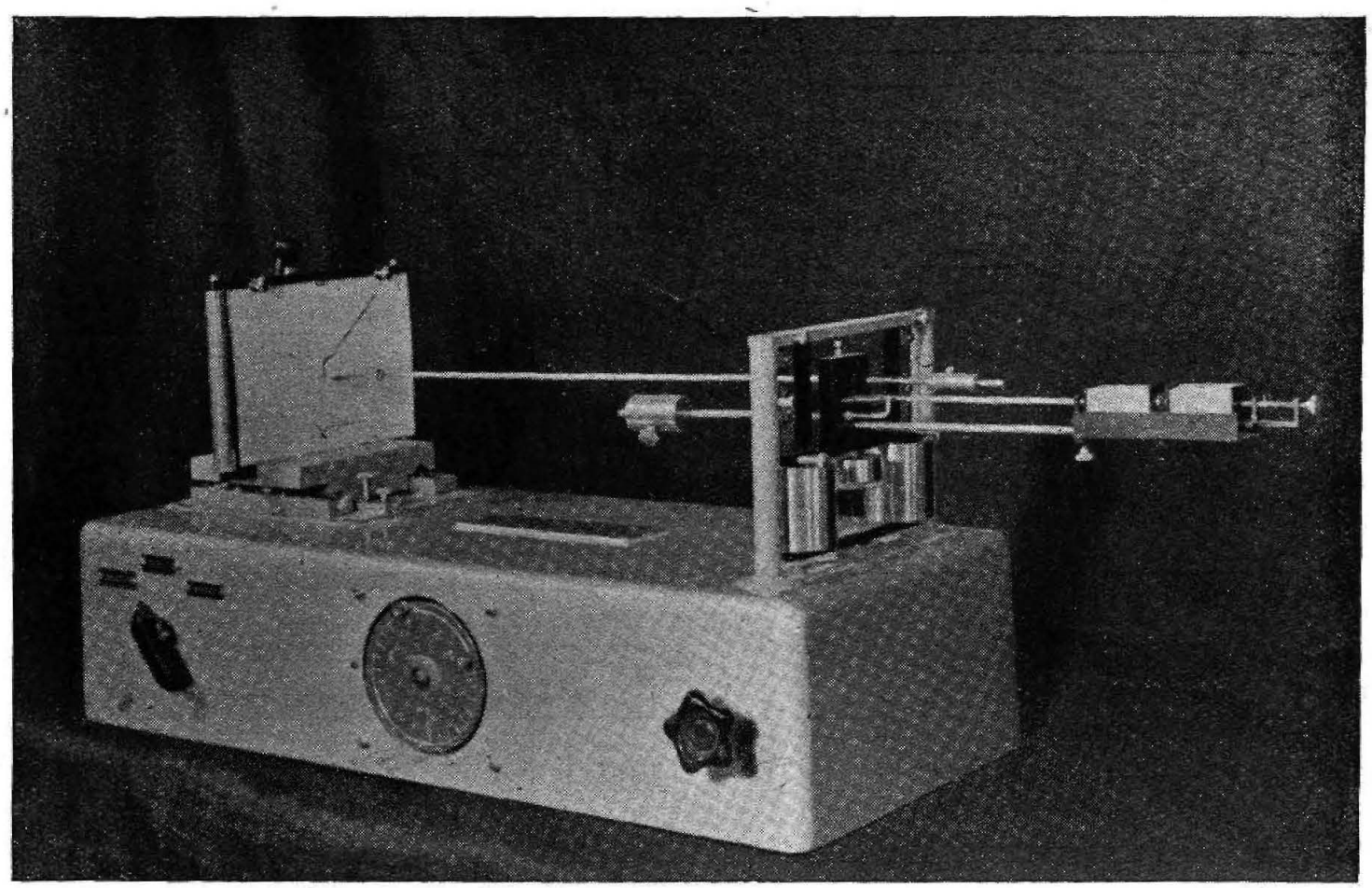

Fig. 2

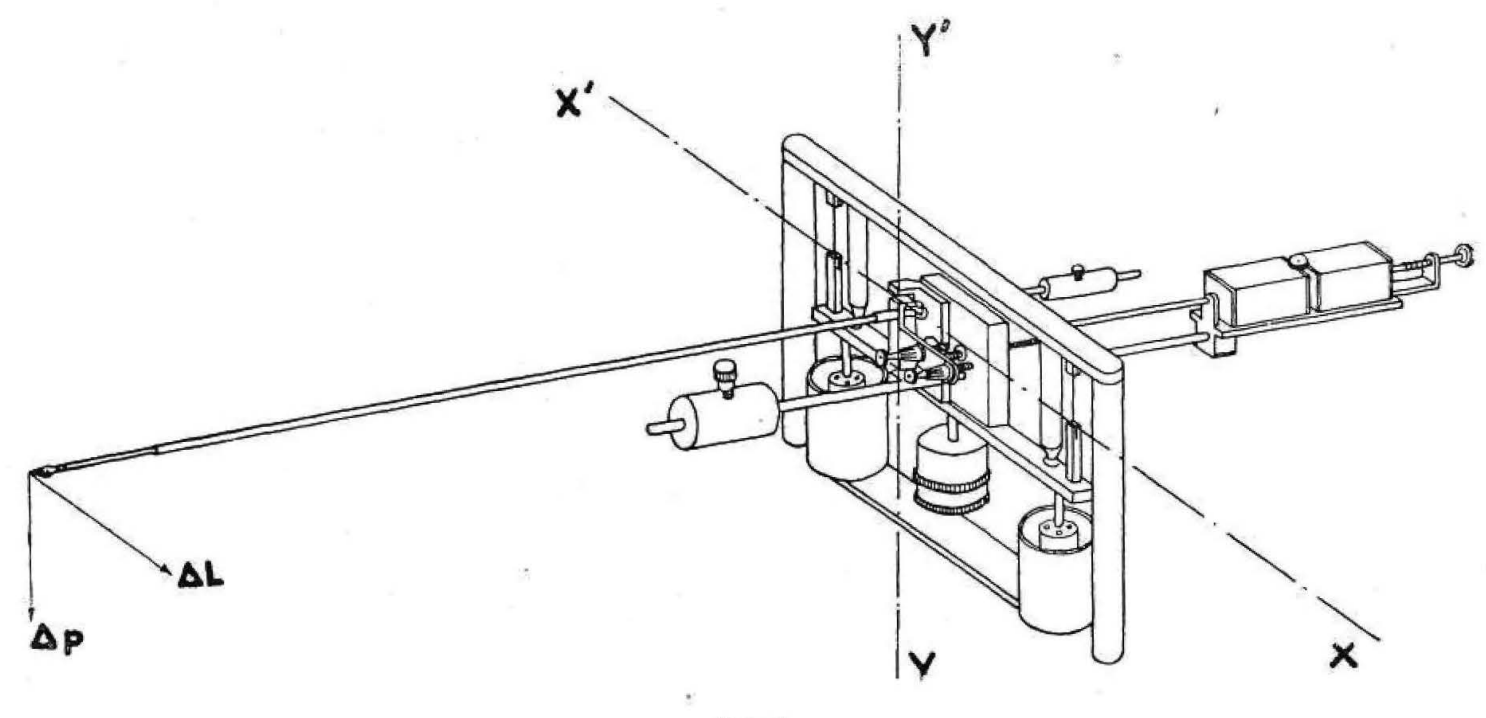

Fig. 3 
Los dos primeros sistemas deben ser proscritos, ya que se obtienen dispersiones enormes.

Los otros dos procedimientos son mucho más exactos, y todavía más el de la galletera que, como es el sistema industrial más extendido, es el que debe usarse.

El trazado de esta curva por puntos, haciendo las medidas un operador, es un trabajo pesado que exige una precisión grande.

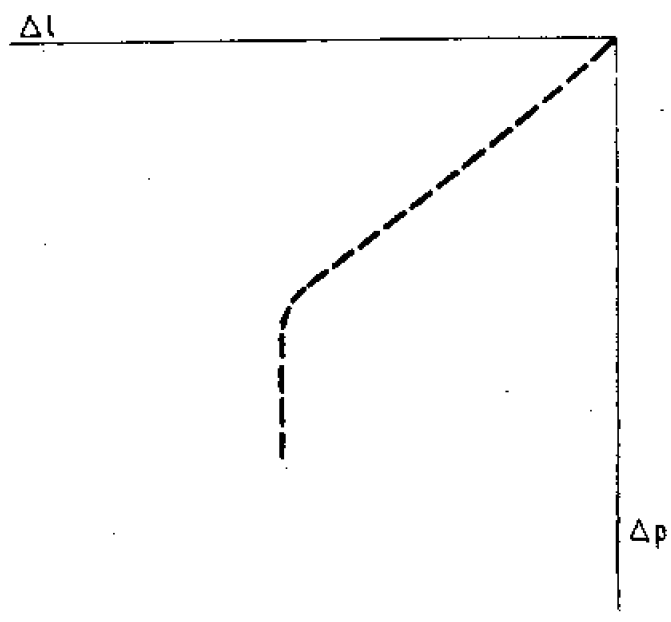

Fig 4

Este problema se ha resuelto mediante el aparato llamado barelatógrafo, que registra la curva automáticamente y evita los errores personales y fatiga del operador. Su conjunto puede verse en la figura 2 .

La figura 3 es un esquema de dicho aparato. Una pluma articulada según dos ejes ortogonales dibuja una curva, la cual depende de la inclinación de la balanza y desviación de una palanca movida por la contracción de la probeta.
La curva se registra sobre un papel, cuyo plano es normal a la dirección media de la aguja. El rozamiento continuo de la pluma sobre el papel se ha eliminado debido a un contacto intermitente entre la pluma y el papel.

Un contactor horario permite interrumpir el trazado de la curva periódicamente, con lo cual se obtiene una curva a trazos. Cada trazo corresponde a una hora $y$, de esta manera, queda también representado en la curva el tiempo.

La figura 4 representa una curva obtenida en nuestro laboratorio con paradas de $15 \mathrm{mi}$ nutos cada 45 de marcha.

Medlante un dispositivo adecuado puede obtenerse también la curva pérdida de pesotiempo.

Se han puesto dos reparos a las curvas de Bigot; uno de ellos tiene por fundamento que. en la representación adoptada por Bigot, se evalúan las humedades con relación a la materia seca, en tanto que las variaciones dimensionales se representan con relación a la longitud tomada en estado húmedo:

Por otra parte, algunos autores afirman que para caracterizar una arcilla no es suficiente una curva de Bigot. Sería necesaria una serie de curvos, en la que cada una correspondería a una humedad inicial diferente.

Hay que hacer notar que una cosa es estudiar el secado industrial de un producto y otra caracterizar una arcilla.

Teniendo en cuenta que el cambio de variable es sencillo, Munier y Gerord-Hirne han dado un procedimiento para pasar de una serie de curvas de Bigot a otra que llama de «contracción absolutass,

En esta última representación cada arcilla se caracteriza por una sola curva, independientemente de la humedad inicial de la probeta.

La comparación de dos arçillas es más fácil estudiarla con una curva para cada una de ellas que con dos series de curvas. 